\title{
Detección de mutaciones asociadas a cepas multidrogo resistente de Mycobacterium tuberculosis en Chile
}

\author{
PAMELA ARAYA ${ }^{1, \mathrm{a}}$, MARITZA VELASCO $^{2, \mathrm{~b}}$, JAVIER TOGNARELLI $^{1, \mathrm{a}}$, \\ FABIOLA ARIAS $^{2, b}$, TAMARA LEIVA ${ }^{2, b}$, ANGÉLICA SCCAPATTICIO ${ }^{2, b}$, \\ PABLO ALVIZ ${ }^{1, a}$, JORGE FERNÁNDEZ ${ }^{1, c}$
}

\section{Detection of genes associated with drug resistance in Mycobacterium tuberculosis strains isolated in Chile}

Background: The incidence of acquired resistance to antituberculous drugs of Mycobacterium tuberculosis in Chile is approximately 23\%. Aim: To analyze the mutations associated with drug resistance in drug- resistant strains of Mycobacterium tuberculosis. Material and Methods: In 28 drug resistant Mycobacterium tuberculosis strains isolated in Chile, genes leading to drug resistance were studied. DNA was amplified by polymerase chain reaction (PCR) and sequencing was carried out using the ABI PRISM big dye terminator cycle sequencing ready reaction kit. Results: In rifampicin-resistant strains, the mutations in rpo $\beta$ gene were in the codons $S 531 \mathrm{~W} / \mathrm{L}$ (56\%), D516Y (16\%) and D516V (16\%). The predominant mutation in kat G gene was in the codon S315L (73\%) in isoniazid-resistant strains. The mutation S95T was found in the $71 \%$ of ciprofloxacin resistant strains. Only one ethambutol-resistant strain had the M306I mutation. Three unreported mutations in kat $G$ were identified. Conclusions: Drug-resistance associated mutations of Mycobacterium tuberculosis isolated in Chile were similar to those reported abroad.

(Rev Med Chile 2011; 139: 467-473).

Key words: Drug resistance, microbial; Mutation; Mycobacterium tuberculosis.

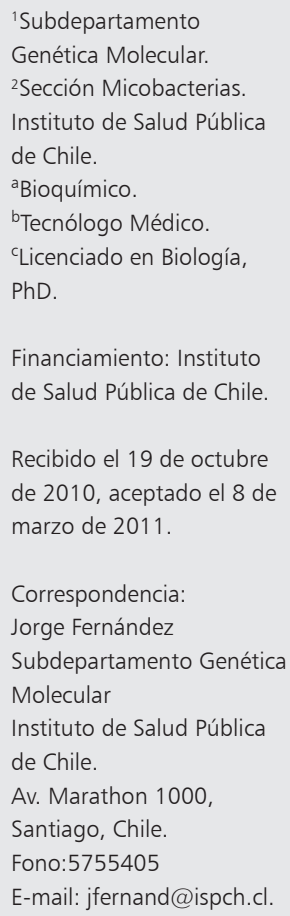

L a incidencia de tuberculosis (TB) se ha incrementado en muchos países durante las últimas décadas y en la actualidad es la enfermedad infecciosa más común en el mundo ${ }^{1}$. Según estimaciones de la Organización Mundial de la Salud en el año 2008 hubo una incidencia de alrededor de 9,4 millones de casos nuevos de tuberculosis en todo el mundo ${ }^{1}$.

La emergencia de cepas resistentes a drogas de TB, especialmente cepas multidrogo resistentes (TB-MDR), ha planteado una amenaza a los programas de control de la tuberculosis. La TB multidrogo resistente es reconocida como una infección con Mycobacterium tuberculosis resistente a las drogas isoniazida (INH) y rifampicina (RIF) de manera simultánea, que son agentes antituber- culosos de primera línea y la columna vertebral del actual tratamiento antituberculosis ${ }^{2,3}$. En 2008, los casos nuevos de TB-MDR se estimaron en 440.000 casos y los casos prevalentes son estimados en sobre un millón ${ }^{1,4}$.

En Chile, la tasa de casos de TB es una de las más bajas en Sudamérica, con una incidencia estimada de 13,2 casos por 100.000 habitantes en el año 2009 (observaciones no publicadas). Sin embargo, la incidencia de tuberculosis en Chile varía según la zona geográfica, con tasas de 20-38 casos por 100.000 habitantes en algunas ciudades en el norte y sur del país ${ }^{5}$. La resistencia adquirida a drogas antituberculosas durante el período 1999 al 2008 ha sido en promedio de $23,4 \%$. Durante los años 2007 y 2008 se observó una resistencia de 
$26,9 \%$ y $22,2 \%$ respectivamente (observaciones no publicadas). La resistencia a rifampicina durante los años 2007 y 2008 fue de 8,4\% y 7,1\% respectivamente. En cambio, la resistencia isoniazida durante el 2007 y 2008 fue de 14,9\% y 11,5\% respectivamente. La multidrogo resistencia durante estos dos años fue de 5,7\% y 4,3\% (observaciones no publicadas).

El análisis genético de las cepas TB-MDR ha revelado diferentes mecanismos de resistencia. $\mathrm{La}$ resistencia a RIF, sobre $90 \%$ de los casos, ha sido asociada con mutaciones en la región principal de 81 pares de bases del gen $r p o \beta$ que codifica para la subunidad- $\beta$ de la ARN polimerasa ${ }^{7-9}$. En contraste, las mutaciones causantes de la resistencia a INH están dispersas en el gen katG (catalasaperoxidasa), la región promotor de $\operatorname{ahpC}$ (alquil hidroperoxidasa), inhA (enoil-acil reductasa), kasA ( $\beta$-ketoacil ACP sintasa), mabA (3-cetoacil reductasa) y $n d h$ (NADH dehidrogenasa) ${ }^{2,10,-14}$. Sin embargo, en 60 a $96 \%$ de los casos, la resistencia a INH es asociada con mutaciones en el gen $\mathrm{kat} G$ principalmente en el codón 315 ( $\mathrm{Ser} \rightarrow \mathrm{Thr})^{9,15-18}$. También se han detectado resistencia a otras drogas antituberculosas, que forman parte del tratamiento que reciben los pacientes. La resistencia a etambutol (EMB) se ha asignado al operón em$b \mathrm{ABC}$, que codifica para la arabinosil transferasa. Mutaciones puntuales en los genes de embABC comúnmente ocurren en embB en el codón 306 ${ }^{19-21}$. Finalmente, en las fluoroquinolonas (FQ), usadas como agentes de retratamiento antituberculosis y recomendadas para tratar las TB-MDR, las mutaciones se ubican en el gen $g y r A^{22}$.

El objetivo de este trabajo fue analizar las mutaciones en los genes rpo $\beta, k a t G$, inh $\mathrm{A}, e m b B$ y gyrA en cepas de Mycobacterium tuberculosis resistentes a las drogas antituberculosas. Este estudio es el primero en describir las mutaciones asociadas a la resistencia a drogas en cepas chilenas de Mycobacterium tuberculosis.

\section{Material y Método}

\section{Cepas}

Las cepas fueron obtenidas de distintos laboratorios de los Servicios de Salud que conforman la red de bacteriología de la tuberculosis en Chile. Dos cepas provenían de la zona norte, dieciocho de la zona central y ocho de la zona sur. Todas las cepas fueron identificadas como M. tuberculosis, por pruebas de identificación fenotípicas estándares. Estas fueron analizadas para determinar la resistencia por el método de las proporciones de Canetti, Rist y Grosset a las concentraciones inhibitorias mínimas correspondientes a cada droga (INH $(0,2 \mu \mathrm{g} / \mathrm{ml})$, RIF $(40 \mu \mathrm{g} / \mathrm{ml})$, CP $(2 \mu \mathrm{g} / \mathrm{ml})$ y $\operatorname{EMB}(2 \mu \mathrm{g} / \mathrm{ml})$. De un total de 28 cepas resistentes, 23 fueron MDR y 5 cepas fueron monoresistentes (Tabla 1). Se utilizaron 10 cepas susceptibles como controles para el mismo período de tiempo. Las cepas de $M$. tuberculosis fueron conservadas a $-70^{\circ} \mathrm{C}$ en medio middlebrook suplementado con OADC/PANTA y mostacillas ${ }^{23}$.

\section{Extracción de ADN}

A partir del cultivo en medio Lowenstein Jensen, se tomó una porción de bacteria con una asa y se resuspendió en $500 \mu \mathrm{l}$ de TE $(10 \mathrm{mM}$ Tris $\mathrm{pH}$ 8,0, $1 \mathrm{mM}$ EDTA). La suspensión fue inactivada por calentamiento a $95^{\circ} \mathrm{C}$ por 15 minutos. El ADN fue aislado por tratamiento con bromuro de cetiltrimetilamonio en presencia de $0,7 \mathrm{M} \mathrm{NaCl}$ como se ha descrito anteriormente ${ }^{24}$.

Amplificación de ADN mediante reacción en cadena de la polimerasa (PCR): Las principales regiones que determinan resistencia en los genes $k a t G$, inh $A, r p o \beta, e m b B$ y gyrA fueron amplificadas usando partidores de oligonucleótidos y condiciones de PCR descritas previamente (Tabla $2)^{21,22,24,26}$. Para la amplificación del ADN se utilizó el termociclador GeneAmp System 2700 (Applied Biosystems). Los productos de PCR fueron purificados con un kit de extracción de gel de Omega Bio-tek (OMEGA, USA) según instrucciones del fabricante.

\section{Secuenciación de ADN}

La secuenciación fue realizada con dideoxinucleótidos marcados con fluorescencia usando el kit "ABI PRISM big dye terminator cycle sequencing ready reaction" (Applied Biosystems, Foster City, CA.) y se utilizaron los mismo partidores con los que se realizó la amplificación por PCR. Las secuencias nucleotídicas fueron analizadas usando el equipo ABI PRISM 3130 Genetic Analyser (Applied Biosystems). Las secuencias fueron ensambladas y editadas con los programas ALIGN, EDITSEQ y MEGALIGN (DNASTAR, Madison, Wis) y fueron comparadas con la secuencia correspondiente de la cepa susceptible de M. tuberculosis. 
Tabla 1. Mutaciones identificadas en las cepas resistentes a drogas antituberculosas

\begin{tabular}{|c|c|c|c|c|c|c|c|}
\hline Aislado & $\begin{array}{c}\text { Natureza } \\
\text { de } \\
\text { resistencia }\end{array}$ & $\begin{array}{l}\text { Patrón de } \\
\text { resistencia } \\
\text { fenotípicab }\end{array}$ & $\begin{array}{l}\text { Cambios } \\
\text { en } r p o \beta\end{array}$ & $\begin{array}{l}\text { Cambios } \\
\text { en katG }\end{array}$ & $\begin{array}{l}\text { Cambios } \\
\text { en inhA }\end{array}$ & $\begin{array}{l}\text { Cambios } \\
\text { en embB }\end{array}$ & $\begin{array}{l}\text { Cambios } \\
\text { en gyrA }\end{array}$ \\
\hline $164-00$ & 2 & $\mathrm{H}^{r}$ & & $\begin{array}{l}\text { 315; AGC } \rightarrow \text { ACC } \\
\text { Se } \rightarrow \text { Thr }\end{array}$ & WT & & \\
\hline $587-00$ & 2 & $\mathrm{H}^{r}$ & & 450; deleción* & WT & & \\
\hline $786-00$ & 2 & $\mathrm{H}^{\mathrm{r}}$ & & $\begin{array}{l}\text { 315; AGC } \rightarrow \text { ACC } \\
\text { Ser } \rightarrow \text { Thr }\end{array}$ & WT & & \\
\hline $8-00$ & 2 & $\mathrm{R}^{\mathrm{r}}$ & $\begin{array}{l}\text { 516; GAC } \rightarrow \text { GTC } \\
\text { Asp } \rightarrow \text { Val }\end{array}$ & & & & \\
\hline $573-00$ & 2 & $\mathrm{R}^{\mathrm{r}}$ & WT & & & & \\
\hline $241-00$ & 2 & $\mathrm{R}^{r}, \mathrm{H}^{r}, \mathrm{Sr}$ & $\begin{array}{l}\text { 531; TCG } \rightarrow \text { TGG } \\
\text { Ser } \rightarrow \text { Trp }\end{array}$ & $\begin{array}{l}\text { 315; AGC } \rightarrow \text { ACC } \\
\text { Ser } \rightarrow \text { Thr }\end{array}$ & WT & & \\
\hline $743-00$ & 1 & $\mathrm{R}^{r}, \mathrm{H}^{\mathrm{r}}$ & $\begin{array}{l}\text { 526; CAC } \rightarrow \text { TAC } \\
\text { His } \rightarrow \text { Tyr }\end{array}$ & $\begin{array}{l}\text { 315; AGC } \rightarrow \text { ACC } \\
\text { Ser } \rightarrow \text { Thr }\end{array}$ & WT & & \\
\hline 277-01 & 2 & $\mathrm{R}^{r}, \mathrm{H}^{r}, \mathrm{~S}^{\mathrm{r}}$ & $\begin{array}{l}\text { 531; TCG } \rightarrow \text { TTG } \\
\text { Ser } \rightarrow \text { Leu }\end{array}$ & 485; deleción* & WT & & \\
\hline $337-02$ & 2 & $\mathrm{R}^{r}, \mathrm{H}^{r}, \mathrm{Sr}$ & $\begin{array}{l}\text { 531; TCG } \rightarrow \text { TTG } \\
\text { Ser } \rightarrow \text { Leu }\end{array}$ & $\begin{array}{l}\text { 315; AGC } \rightarrow \text { ACC } \\
\text { Ser } \rightarrow \text { Thr }\end{array}$ & WT & & \\
\hline $502-00$ & 2 & $\mathrm{R}^{r}, \mathrm{H}^{r}, \mathrm{~S}^{\mathrm{r}}$ & $\begin{array}{l}\text { 531; TCG } \rightarrow \text { TTG } \\
\text { Ser } \rightarrow \text { Leu }\end{array}$ & $\begin{array}{l}\text { 315; AGC } \rightarrow \text { ACC } \\
\text { Ser } \rightarrow \text { Thr }\end{array}$ & WT & & \\
\hline $148-01$ & 2 & $\mathrm{R}^{r}, \mathrm{H}^{r}, \mathrm{~S}^{\mathrm{r}}$ & $\begin{array}{l}531 ; \text { TCG } \rightarrow \text { TGG } \\
\text { Ser } \rightarrow \text { Trp }\end{array}$ & WT & WT & & \\
\hline 490-02 & 1 & $\mathrm{R}^{r}, \mathrm{H}^{r}, \mathrm{~S}^{r}$ & $\begin{array}{l}\text { 531; TCG } \rightarrow \text { TTG } \\
\text { Ser } \rightarrow \text { Leu }\end{array}$ & $\begin{array}{l}\text { 315; AGC } \rightarrow \text { ACC } \\
\text { Ser } \rightarrow \text { Thr }\end{array}$ & WT & & \\
\hline $372-02$ & 2 & $\mathrm{Rr}, \mathrm{H}^{r}, \mathrm{~S}^{\mathrm{r}}$ & $\begin{array}{l}\text { 531; TCG } \rightarrow \text { TTG } \\
\text { Ser } \rightarrow \text { Leu }\end{array}$ & $\begin{array}{l}\text { 381; GAC } \rightarrow \text { TAC } \\
\text { Asp } \rightarrow \text { Tyr }\end{array}$ & WT & & \\
\hline $392-01$ & 2 & $\mathrm{R}^{r}, \mathrm{H}^{r}, \mathrm{~S}^{\mathrm{r}}$ & $\begin{array}{l}\text { 526; CAC } \rightarrow \text { TAC } \\
\text { His } \rightarrow \text { Tyr }\end{array}$ & $\begin{array}{l}\text { 315; AGC } \rightarrow \text { ACC } \\
\text { Ser } \rightarrow \text { Thr }\end{array}$ & WT & & \\
\hline 714-01 & 2 & $\mathrm{R}^{r}, \mathrm{H}^{r}, \mathrm{~S}^{\mathrm{r}}$ & $\begin{array}{l}\text { 516; GAC } \rightarrow \text { GTC } \\
\text { Asp } \rightarrow \text { Val }\end{array}$ & $\begin{array}{l}\text { 315; AGC } \rightarrow \text { ACC } \\
\text { Ser } \rightarrow \text { Thr }\end{array}$ & WT & & \\
\hline $318-00$ & 2 & $\mathrm{R}^{r}, \mathrm{H}^{r}, \mathrm{~S}^{r}$ & WT & $\begin{array}{l}\text { 315; AGC } \rightarrow \text { ACC } \\
\text { Ser } \rightarrow \text { Thr }\end{array}$ & WT & & \\
\hline $792-00$ & 2 & $\mathrm{R}^{r}, \mathrm{H}^{\mathrm{r}}, \mathrm{S}^{\mathrm{r}}$ & $\begin{array}{l}531 ; \text { TCG } \rightarrow \text { TGG } \\
\text { Ser } \rightarrow \text { Trp }\end{array}$ & $\begin{array}{l}\text { 315; AGC } \rightarrow \text { ACC } \\
\text { Ser } \rightarrow \text { Thr }\end{array}$ & WT & & \\
\hline $574-98$ & 2 & $\mathrm{R}^{r}, \mathrm{H}^{r}, \mathrm{~S}^{r}$ & $\begin{array}{l}531 ; \text { TCG } \rightarrow \text { TGG } \\
\text { Ser } \rightarrow \text { Trp }\end{array}$ & $\begin{array}{l}\text { 315; AGC } \rightarrow \text { ACC } \\
\text { Ser } \rightarrow \text { Thr }\end{array}$ & WT & & \\
\hline $137-01$ & 2 & $\mathrm{R}^{r}, \mathrm{H}^{r}, \mathrm{Sr}, \mathrm{CPr}$ & $\begin{array}{l}\text { 531; TCG } \rightarrow \text { TTG } \\
\text { Ser } \rightarrow \text { Leu }\end{array}$ & $\begin{array}{l}\text { 315; AGC } \rightarrow \text { ACC } \\
\text { Ser } \rightarrow \text { Thr }\end{array}$ & С59T & & $\begin{array}{l}\text { 95; AGC } \rightarrow \text { ACC } \\
\text { Ser } \rightarrow \text { Thr }\end{array}$ \\
\hline $524-99$ & 2 & $\mathrm{R}^{r}, \mathrm{H}^{r}, \mathrm{CP}^{r}$ & $\begin{array}{l}\text { 526; CAC } \rightarrow \text { TAC } \\
\text { His } \rightarrow \text { Tyr }\end{array}$ & $\begin{array}{l}\text { 315; AGC } \rightarrow \text { ACC } \\
\text { Ser } \rightarrow \text { Thr }\end{array}$ & WT & & WT \\
\hline $259-00$ & 2 & $\mathrm{R}^{r}, \mathrm{H}^{r}, \mathrm{CPr}, \mathrm{E}^{\mathrm{r}}$ & $\begin{array}{l}531 ; \mathrm{TCG} \rightarrow \mathrm{TGG} \\
\mathrm{Ser} \rightarrow \operatorname{Trp}\end{array}$ & $\begin{array}{l}\text { 315; AGC } \rightarrow \text { ACC } \\
\text { Ser } \rightarrow \text { Thr }\end{array}$ & WT & WT & $\begin{array}{l}\text { 95; AGC } \rightarrow \text { ACC } \\
\text { Ser } \rightarrow \text { Thr }\end{array}$ \\
\hline $645-00$ & 2 & $\mathrm{R}^{r}, \mathrm{H}^{\mathrm{r}}, \mathrm{CP}, \mathrm{E}^{\mathrm{r}}$ & $\begin{array}{l}\text { 516; GAC } \rightarrow \text { GTC } \\
\text { Asp } \rightarrow \text { Val }\end{array}$ & $\begin{array}{l}\text { 344; ACG } \rightarrow \text { CCG } \\
\text { Thr } \rightarrow \text { Pro* }\end{array}$ & WT & WT & $\begin{array}{l}\text { 95; AGC } \rightarrow \text { ACC } \\
\text { Ser } \rightarrow \text { Thr }\end{array}$ \\
\hline
\end{tabular}


Tabla 1. Mutaciones identificadas en las cepas resistentes a drogas antituberculosas (continuación)

\begin{tabular}{|c|c|c|c|c|c|c|c|}
\hline Aislado & $\begin{array}{c}\text { Natureza } \\
\text { de } \\
\text { resistencia }^{a}\end{array}$ & $\begin{array}{l}\text { Patrón de } \\
\text { resistencia } \\
\text { fenotípicab }^{\text {benotion }}\end{array}$ & $\begin{array}{l}\text { Cambios } \\
\text { en } r p o \beta\end{array}$ & $\begin{array}{l}\text { Cambios } \\
\text { en katG }\end{array}$ & $\begin{array}{l}\text { Cambios } \\
\text { en inhA }\end{array}$ & $\begin{array}{l}\text { Cambios } \\
\text { en embB }\end{array}$ & $\begin{array}{l}\text { Cambios } \\
\text { en gyrA }\end{array}$ \\
\hline $526-01$ & 2 & $\mathrm{R}^{r}, \mathrm{H}^{r}, \mathrm{~S}^{r} \mathrm{E}^{\mathrm{r}}$ & $\begin{array}{l}\text { 526; CAC } \rightarrow \text { TAC } \\
\text { His } \rightarrow \text { Tyr }\end{array}$ & $\begin{array}{l}\text { 315; AGC } \rightarrow \text { ACC } \\
\text { Ser } \rightarrow \text { Thr }\end{array}$ & WT & WT & \\
\hline $319-00$ & 2 & $\mathrm{R}^{r}, \mathrm{H}^{r}, \mathrm{CP}^{r} \mathrm{E}^{\mathrm{r}}$ & $\begin{array}{l}531 ; \text { TCG } \rightarrow \text { TGG } \\
\text { Ser } \rightarrow \text { Trp }\end{array}$ & $\begin{array}{l}\text { 315; AGC } \rightarrow \text { ACG } \\
\text { Ser } \rightarrow \text { Thr } \\
\text { 463; CGG } \rightarrow \text { CTG } \\
\text { Arg } \rightarrow \text { Leu }\end{array}$ & WT & WT & $\begin{array}{l}\text { 95; AGC } \rightarrow \text { ACC } \\
\text { Ser } \rightarrow \text { Thr }\end{array}$ \\
\hline $702-01$ & 2 & $\begin{array}{l}\mathrm{Rr}^{r}, \mathrm{H}^{r}, \mathrm{~S}^{\mathrm{r}} \\
\mathrm{CP}^{r}, \mathrm{E}^{\mathrm{r}}\end{array}$ & $\begin{array}{l}\text { 531; TCG } \rightarrow \text { TTG } \\
\text { Ser } \rightarrow \text { Leu }\end{array}$ & WT & С59T & WT & $\begin{array}{l}\text { 95; AGC } \rightarrow \text { ACC } \\
\text { Ser } \rightarrow \text { Thr }\end{array}$ \\
\hline $138-01$ & 2 & $\begin{array}{l}\mathrm{R}^{r}, \mathrm{H}^{r}, \mathrm{~S}^{\mathrm{r}} \\
\mathrm{CP}, \mathrm{E}^{\mathrm{r}}\end{array}$ & $\begin{array}{l}\text { 516; GAC } \rightarrow \text { GTC } \\
\text { Asp } \rightarrow \text { Val }\end{array}$ & $\begin{array}{l}\text { 315; AGC } \rightarrow \text { ACC } \\
\text { Ser } \rightarrow \text { Thr }\end{array}$ & WT & WT & WT \\
\hline 715-01 & 2 & $\mathrm{R}^{r}, \mathrm{H}^{r}, \mathrm{~S}^{r}, \mathrm{E}^{\mathrm{r}}$ & $\begin{array}{l}531 ; \text { TCG } \rightarrow \text { TGG } \\
\text { Ser } \rightarrow \text { Trp }\end{array}$ & WT & WT & M306I & \\
\hline $337 b-01$ & 1 & $\mathrm{R}^{r}, \mathrm{H}^{r}, \mathrm{~S}^{r} \mathrm{E}^{\mathrm{r}}$ & WT & $\begin{array}{l}\text { 315; AGC } \rightarrow \text { ACC } \\
\text { Ser } \rightarrow \text { Thr }\end{array}$ & WT & WT & \\
\hline
\end{tabular}

Mutaciones de las resistencia más comunes de las regiones de katG (620 pb, codón 300-505), promotor inhA (248 pb,-199 $a+47), r p o \beta(210$ pb, codón 493-539), embB (461 pb, codón 268-420) y gyrA (320 pb, codón 35-141). Se muestran los cambios de nucleótidos y aminoácidos. a: 1, Resistencia primaria; 2, resistencia secundaria. ${ }^{b}$ : Rr resistente a rifampicina $\mathrm{H}^{r}$, resistencia a isoniazida; $\mathrm{S}^{r}$, resistencia a estreptomicina; $\mathrm{CP}^{r}$, resistente a ciprofloxacino; $\mathrm{E}^{\mathrm{r}}$ resistente a etambutol. Asteriscos indican mutaciones nuevas; WT, tipo silvestre (Wild-type).

Tabla 2. Oligonucleótidos utilizados para la amplificación de los genes de resistencia

\begin{tabular}{|c|c|c|c|}
\hline Genes/(referencia) & Secuencia nucleotídica & TM $\left({ }^{\circ} \mathrm{C}\right)$ & $\begin{array}{l}\text { Tamaño amplicón } \\
\text { (pb) }\end{array}$ \\
\hline$r p o \beta(26)$ & $\begin{array}{l}5^{\prime} \text { cgt gga ggc gat cac acc gca gac gt } 3^{\prime} \\
5^{\prime} \text { agt gcg acg ggt gca cgt gcg gga cct } 3^{\prime}\end{array}$ & 61 & 215 \\
\hline katG (26) & $\begin{array}{l}5^{\prime} \text { agc tcg tat ggc acc gga ac } 3^{\prime} \\
5^{\prime} \text { ttg acc tcc cac ccg act tg } 3^{\prime}\end{array}$ & 60 & 620 \\
\hline $\operatorname{inh} A(11)$ & $\begin{array}{l}5^{\prime} \text { cct cgc tgc cca gaa agg ga3' } \\
5^{\prime} \text { atc ccc cgg ttt cct ccg gt } 3^{\prime}\end{array}$ & 55 & 248 \\
\hline embB (21) & $\begin{array}{l}5^{\prime} \text { aat tgc cca gct cct cct cag } 3^{\prime} \\
5^{\prime} \text { aca gca gca gcc agc aca cta } 3^{\prime}\end{array}$ & 60 & 361 \\
\hline gyrA (11) & $\begin{array}{l}5^{\prime} \text { cag cta cat cga cta tgc ga } 3 \\
5^{\prime} \text { ggg ctt cgg tgt acc tca t } 3^{\prime}\end{array}$ & 45 & 320 \\
\hline
\end{tabular}

\section{Resultados}

En 28 cepas de Mycobacterium tuberculosis resistentes a drogas se analizaron los genes asociados a la resistencia mediante secuenciamiento genético. Las cepas fueron originalmente cultivadas entre los años 1998 al 2001 y provenían de diferentes zonas geográficas de Chile. Se estableció que 25 cepas provenían de pacientes con un historial de tratamiento previo y 3 cepas correspondieron a pacientes sin tratamiento previo (Tabla 1 ).

En $22(88 \%)$ de las 25 cepas resistentes a rifampicina se identificaron mutaciones en los codones: 516 ( 4 cepas), 526 ( 4 cepas) y 531 (14 cepas) (Tabla 1). En el codón 531 se encontraron las mutaciones $\mathrm{TCG} \rightarrow$ TTG $(\mathrm{S} 531 \mathrm{~L})$ y TCG $\rightarrow$ TGG $(\mathrm{S} 531 \mathrm{~W})$ que 
produjo la sustitución del aminoácido serina a leucina y de serina a triptófano en este codón respectivamente. Las mutaciones en los codones 516 y 526 produjeron la sustitución de ácido aspártico a valina y de histidina a tirosina respectivamente. En ninguna de las cepas susceptibles se encontraron mutaciones a RIF.

Las mutaciones en kat $G$ fueron detectadas en 23 de los 26 cepas resistentes (88,5\%), pero en ninguna de las 10 cepas susceptibles. En 20 cepas se encontró una mutación puntual y en 2 cepas se detectó una deleción de 3 bases (Tabla 1). La mutación más común correspondió al codón 315 de $k a t G$, en 18 cepas se identificó la mutación AGC $\rightarrow$ ACC y 1 cepa la mutación AGC $\rightarrow$ ACG. Ambas mutaciones producen la sustitución de serina por treonina en este codón. Otras dos mutaciones fueron detectadas en los codones 381 y 463 del gen $k a t G$. En tres cepas MDR no se detectaron mutaciones en la región del gen $k a t G$ estudiado. En la región promotora del gen inhA se identificó la mutación C59T en 2 cepas.

En una cepa resistente a EMB se encontró una mutación en el codón 306 generando un cambio de metionina a isoleucina. En las cepas susceptibles no se encontraron mutación en la región $e m b B$.

En las siete cepas resistentes a ciprofloxacina (CP) se analizó el gen gyrA, en $5(71,4 \%)$ de ellas se encontró la mutación S95T.

\section{Discusión}

Los mecanismos de resistencia a RIF están basados en cambios estructurales de la ARN polimerasa, sugiriendo que la región de la subunidad $\beta$ de la enzima juega un rol importante en determinar el fenotipo de resistencia ${ }^{15}$. Mutaciones en uno de los 3 codones (516, 526 y 531) se observan para la mayoría de los aislados resistentes a RIF (70 a 95\%), especialmente en áreas con alta incidencia de MDR-TB ${ }^{7}$. Estudios previos demuestran que la mutación S531L fue la más frecuente ${ }^{18,27}$. En ninguna de las cepas resistentes a RIF se detectaron mutaciones simultaneas en más de un codón y tampoco se encontraron inserciones en los codones 514 y 521 como se ha descrito ${ }^{27,29}$. En 3 cepas resistentes a RIF no se detectaron mutaciones en la región del gen rpoß que se estudió. Este resultado indica que la resistencia a RIF en estas cepas, podría estar relacionado con mutaciones en otra región del gen rpo $\beta$ o que otro mecanismo diferente es responsable de esta resistencia ${ }^{30}$.

En las cepas resistentes a INH, se observó principalmente la mutación en el codón 315 de $k a t G$, como se ha reportado previamente ${ }^{14,18}$. Las mutaciones en los codones 381 y 463 también han sido reportadas, sin embargo, se ha descrito que la mutación R463L (CGG $\rightarrow$ CTG) es un polimorfismo que no influencia la resistencia a INH ${ }^{18,31,32,33}$. En este estudio se encontraron 3 mutaciones en el gen $k a t G$ que no han sido descritas en la literatura (T344P y las deleciones de 3 bases en los codones 450 y 485). Sin embargo, desconocemos si estas mutaciones están asociadas a la resistencia a INH.

Estudios previos han identificados otros genes responsables de la resistencia a INH, como los genes ahpC, kasA y $n d h^{13,29}$. Las mutaciones en inhA o su promotor pueden presentarse por sí solas o en combinación con las mutaciones en $k a t G$, lo cual fue observado en una de las cepas resistentes a INH ${ }^{11}$. Por lo tanto, las cepas resistentes a INH que no presentaron mutaciones en $k a t G$ o en inh $A$ la resistencia pueden estar asociadas a otro gen o a otro mecanismo de resistencia.

Se ha encontrado que la resistencia a etambutol genera mutaciones puntuales en los genes embA$B C$, especialmente en el codón 306 de embB $B^{21,26}$. Sin embargo, esta mutación también se ha identificado en cepas susceptibles a este fármaco ${ }^{21}$. Estos resultados pueden sugerir que otro mecanismo esté operando, el cual podría explicar la resistencia a EMB en los aislados de M. tuberculosis con las regiones de $e m b \mathrm{~B}$ intactas ${ }^{26}$.

Las fluoroquinolonas comprenden un régimen de droga para retratamiento en la terapia de TB. La mutación más común en las cepas $\mathrm{CP}$ resistentes fue en el codón S95T, que se ha reportado que no tiene un rol directo en el desarrollo de la resistencia a esta droga, ya que también se ha detectado en cepas sensibles al fármaco ${ }^{22}$. La resistencia a ésta u otra droga antituberculosa podrían deberse a una disminución en la permeabilidad de la pared celular o bien a mecanismos de bombas de eflujo de drogas activas, secuestro e inactivación de la $\operatorname{droga}^{34}$.

El método de las proporciones define una concentración crítica que permite detectar las cepas resistentes a diversas drogas antituberculosas. Los estudios realizados para correlacionar la resistencia fenotípica a rifampicina $(40 \mu \mathrm{g} / \mathrm{ml})$ e isoniazida $(0,2 \mu \mathrm{g} / \mathrm{ml})$ por el método de las proporciones 
con mutaciones en los genes $r p o \beta$ (codones 516, 526 y 531), y katG (codón 315) han demostrado una correlación de $70-95 \%$ para la resistencia a rifampicina y de $42-93 \%$ para la resistencia a isoniazida ${ }^{7-9,15-18}$ en estos codones. Nuestros resultados demostraron una correlación similar entre la resistencia genotípica a rifampicina e isoniazida y las mutaciones encontradas en los genes rpo $\beta$ y kat $G$ en las cepas chilenas de $M$. tuberculosis.

$\mathrm{La}$ alta prevalencia de las mutaciones en la región de rpo $\beta$ así como las mutaciones en el codón 315 de $k a t G$ en las cepas MDR-TB de Chile, indican el potencial que podrían tener las pruebas de diagnóstico rápido para la detección de la resistencia a estas drogas en $M$. tuberculosis. Sin embargo, no se puede descartar que cepas que no posean estas mutaciones sean resistentes a estos fármacos.

Este estudio provee datos valiosos, ya que incrementan nuestro entendimiento de los mecanismos moleculares de la resistencia a drogas y orientan respecto al uso cuidadoso de las técnicas de susceptibilidad a drogas, basadas en la detección de diferentes tipos de mutaciones que ocurren en las cepas chilenas de M. tuberculosis.

Agradecimientos: A María Ibáñez y Luis Sánchez del Subdepartamento Genética Molecular por el apoyo técnico prestado en la ejecución de este trabajo.

\section{Referencias}

1. Global tuberculosis control: WHO report 2010. Geneva, World Health Organization (WHO/HTM/TB/2010.7).

2. Leung ET, Ho PL, Yuen KY, Woo WL, Lam TH, Kao RY, et al. Molecular characterization of isoniazid resistance in Mycobacterium tuberculosis: identification of a novel mutation in inhA. Antimicrob Agents Chemother 2006; 50: $1075-8$.

3. Dahle U, Sanven P, Heldal E, Mannsaaker T, Caugant D. Deciphering an outbreak of Drug-resistant Mycobacterium tuberculosis. J Clin Microbiol 2003; 41: 67-72.

4. Matteelli A, Migliori GB, Cirillo D, Centis R, Girard E, Raviglion M. Multidrug-resistant and extensively drugresistant Mycobacterium tuberculosis: epidemiology and control. Expert Rev Anti Infect Ther 2007; 5: 857-71.

5. Minsal.cl. Programa Nacional del Control de la Tuberculosis. Manual de organización y normas técnicas 2005. Available from: http://www.redsalud.gov.cl/archivos/ tuberculosis.

6. Riquelme MC, Velasco M, Rodríguez L. Actualización de la resistencia a drogas antituberculosas en Chile, 2006. Rev Enf Respir 2008; 24; 60-5.

7. Telenti A, Imboden P, Marchesi F, Lowrie D, Cole S, Colston MJ, et al. Detection of rifampicin-resistance mutations in Mycobacterium tuberculosis. Lancet 1993; 341: 647-50.

8. Bartfai Z, Somoskövi A, Ködmön C, Szabo N, Puskas E, Kosztolanyi L, et al. Molecular characterization of rifampin-resistant isolates of Mycobacterium tuberculosis from Hungary by DNA sequencing and the line probe assay. J Clin Microbiol. 2001; 39: 3736-9.

9. Nikolayevsky V, Brown T, Balabanova Y, Ruddy M, Fedorin I, Drobniewski F. Detection of mutations associated with isoniazid and rifampin resistance in Mycobacterium tuberculosis isolates from Samara Region, Russian Federation. J Clin Microbiol 2004; 42(10): 4498-502.

10. Mokrousov I, Narvskaya O, Otten T, Limeschenko E, Steklova L, Vyshnevskiy B. High prevalence of KatG Ser315Thr substitution among isoniazid-resistant Mycobacterium tuberculosis clinical isolates from Northwestern Russia, 1996 to 2001. Antimicrob. Agents Chemother. 2002; 46: 1417-24.

11. Musser JM, Kapur V, Williams DL, Kreiswirth BN, Van Soolingen D, Van Embden JD. Characterization of the catalase-peroxidase gene $k a t G$ and inhA locus in isoniazid-resistant and -susceptible strains of Mycobacterium tuberculosis by automated DNA sequencing: restricted array of mutations associated with drug resistance. J Infect Dis 1996; 173: 196-202.

12. Telenti A, Honore N, Bernasconi C, March J, Ortega A, Takiff HE, et al. Genotyping assessment of isoniazid and rifampin resistance in Mycobacterium tuberculosis: a blind study at reference laboratory level. J Clin Microbiol 1997; 35: 719-23.

13. Kelley CL, Rouse DA, Morris SL. Analysis of $a h p C$ gene mutations in isoniazid-resistant clinical isolates of $\mathrm{My}$ cobacterium tuberculosis. Antimicrob Agents Chemother. 1997; 41: 2057-8.

14. Hazbon $\mathrm{MH}$, Brimacombe $\mathrm{M}$, Bobadilla del Valle $\mathrm{M}$, Cavatore M, Guerrero MI, et al. Population genetics study of isoniazid resistance mutations and evolution of multidrug-resistant Mycobacterium tuberculosis. Antimicrob Agents Chemother. 2006; 50: 2640-9.

15. Marttila HJ, Soini H, Eerola E, Vyshnevskaya E, Vyshnevskiy BI, et al. A Ser315Thr substitution in Kat G is predominant in genetically heterogeneous multidrugresistant Mycobacterium tuberculosis isolates originating from the St Petersburg area in Russia. Antimicrob Agents Chemother 1998; 42: 2443-5.

16. Van Soolingen D, De Haas PE, Van Doorn HR, Kuijper E, Rinder $\mathrm{H}$, et al. Mutations at amino acid position 315 
of the kat $\mathrm{G}$ gene are associated with high-level resistance to isoniazid, other drug resistance, and successful transmission of Mycobacterium tuberculosis in the Netherlands. J Infect Dis 2000; 182: 1788-90.

17. Tracevska T, Jansone I, Broka L, Marga O, Baumanis V. Mutations in the $r p o \beta$ and $k a t G$ genes leading to drug resistance in Mycobacterium tuberculosis in Latvia. J Clin Microbiol 2002; 40: 3789-92.

18. Ramaswamy S, Dou S, Rendon A, Yang Z, Cave D, Graviss E. Genotypic analysis of multidrug-resistant $\mathrm{Myco-}$ bacterium tuberculosis isolates from Monterrey, Mexico. J Med Microbiolo 2004; 53; 107-13.

19. Alcaide F, Pfyffer G, Telenti A. Role of embB innatural and acquired resistance to ethambutol in mycobacteria. Antimicrob Agents Chemother 1997; 41: 2270-3.

20. Ramaswamy S, Amin A, Göksel S, Stager C, Dou S, Sahly H, et al. Molecular genetic analysis of nucleotide polymorphisms associated with ethambutol resistance in human isolates of Mycobacterium tuberculosis. Antimicrob Agents Chemother. 2000; 44: 326-36.

21. Shen X, Shen GM, Wu J, Gui XH, Li X, Mei J, et al. Association between embB codon 306 mutations and drug resistance in Mycobacterium tuberculosis. Antimicrob Agents Chemother 2007; 51: 2618-20.

22. Giannoni F, Iona E, Sementilli F, Brunori L, Pardini M, Battista G, et al. Evaluation of a new lineprobe assay for rapid identification of gyrA mutations in Mycobacterium tuberculosis. Antimicrob. agents Chemother 2005; 49: 2928-33.

23. Ministerio de Salud de Brasil. Manual Nacional de Vigilancia de laboratorios de tuberculosis y otras micobacterias. 2008. p. 393-403.

24. Van Embden JDA, Crawford JT, Dale JW, Gicquel B, Hermans P, McAdam R, et al. Strain identification of Mycobacterium tuberculosis by DNA fingerprinting: recommendations for a standardized methodology. J Clin Microbiol 1993; 31; 406-9.

25. Siddiqi N, Shamim M, Hussain S, Choudhary K, Ahmed N, Prachee G, et al. Molecular characterization of multidrug-resistant isolates of Mycobacterium tuberculosis from patients in North India. Antimicrob Agents Chemother 2002; 46: 443-50.

26. Persing DH. PCR protocols for emerging infectious disease. American Society for Microbiology, Washington, 1996. p. 114-49.

27. Lee H, Myoung H, Bang H, Bai G, Kim S, Kim J, et al. Mutations in the $e m b B$ locus among Korean clinical isolates of mycobacterium tuberculosis resistant to etambutol. Yonsei Med J 2002; 43: 59-64.

28. Caws M, Duy PM, Tho DQ, Lan NT, Hoa DV, Farrar J. Mutations prevalent among rifampin- and isoniazidresistant Mycobacterium tuberculosis isolates from a hospital in Vietnam. J Clin Microbiol 2006; 44: 2333-7.

29. Hillemann D, Weizenegger M, Kubica T, Ritchter E, Niemann S. Use of the genotypeMTBDR assay for rapid detection of rifampin and isoniazida resistance in $\mathrm{Myco}$ bacterium tuberculosis complex isolates. J Clin Microbiol 200; 543: 3699-703.

30. Heep M, Brandstätter B, Rieger U, Lehn N, Richter E, Rüsch-Gerdes $S$, et al. Frequency of rpo $\beta$ mutations inside and outside the cluster I region in rifampinresistant clinical Mycobacterium tuberculosis isolates. J Clin Microbiol. 2001; 39: 107-10.

31. Zaker Bostanabad S, Titov LP, Slizen VV, Taghikhani $\mathrm{M}$, Bahrmand A. kat $G$ mutations in isoniazid-resistant strains of Mycobacterium tuberculosis isolates from Belarusian patients. Tuberk Toraks 2007; 55: 231-7.

32. Rouse D, De Vito J, Li Z, Byer H, Morris S. Site-directed mutagenesis of the kat $G$ gene of Mycobacterium tuberculosis: effects on catalasa-oeroxidase activities and isoniazid resistance. Mol Microbio 1996; 22: 583-92.

33. Sekiguchi J, Miyoshi-Akiyama T, Augustynowicz-Kopec E, Zwolska Z, Kirikae F, Toyota E, et al. Detection of multidrug resistance in Mycobacterium tuberculosis. J Clin Microbiol 2007; 45: 179-92.

34. Ginsburg AS, Grosset JH, Bishai WR. Fluoroquinolones, tuberculosis and resistance. Lancet Infect Dis 2003; 3: 432-42. 


\section{Referencias}

1. Martin W, Brynes S. Chilean Miners and Biomedical Research. A Modest Proposal. Am J Respir Crit Care Med 2010; 182: 1459-64.

2. Sobradillo P, Pozo F, Agustí A. Medicina P4: el futuro a la vuelta de la esquina. Arch Bronconeumol 2011; 47: 35-40.

Correspondencia a: Dr. Rafael Silva O.

Dos Norte 360, Talca. Fono: 71-412716 Fax: 71-209306

E-mail: rafaelsilvao@gmail.com 